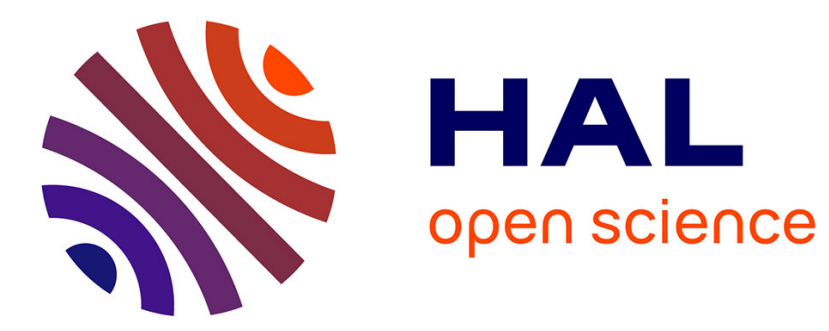

\title{
Rules of Thumb to Assess Losses of Implanted Antennas
} Mingxiang Gao, Denys Nikolayev, Marko Bosiljevac, Zvonimir Sipus, Anja K.

Skrivervik

\section{To cite this version:}

Mingxiang Gao, Denys Nikolayev, Marko Bosiljevac, Zvonimir Sipus, Anja K. Skrivervik. Rules of Thumb to Assess Losses of Implanted Antennas. 15th European Conference on Antennas and Propagation (EuCAP), Mar 2021, Dusseldorf, Germany. 10.23919/EuCAP51087.2021.9411344 . hal03330246

\section{HAL Id: hal-03330246 \\ https://hal.science/hal-03330246}

Submitted on 19 Oct 2021

HAL is a multi-disciplinary open access archive for the deposit and dissemination of scientific research documents, whether they are published or not. The documents may come from teaching and research institutions in France or abroad, or from public or private research centers.
L'archive ouverte pluridisciplinaire HAL, est destinée au dépôt et à la diffusion de documents scientifiques de niveau recherche, publiés ou non, émanant des établissements d'enseignement et de recherche français ou étrangers, des laboratoires publics ou privés. 


\title{
Rules of Thumb to Assess Losses of Implanted Antennas
}

\author{
Mingxiang Gao ${ }^{1}$, Denys Nikolayev ${ }^{2}$, Marko Bosiljevac ${ }^{3}$, Zvonimir Šipuš ${ }^{3}$, Anja K. Skrivervik ${ }^{1}$ \\ ${ }^{1}$ Microwaves and Antennas Group, École Polytechnique Fédérale de Lausanne, Lausanne, Switzerland \\ ${ }^{2}$ IETR (l'Institut d'électronique et des technologies du numérique) UMR 6164, CNRS / Univ. Rennes, Rennes, France \\ ${ }^{3}$ Faculty of Electrical Engineering and Computing, University of Zagreb, Zagreb, Croatia \\ mingxiang.gao@epfl.ch,d@deniq.com,marko.bosiljevac@fer.hr, zvonimir.sipus@fer.hr, anja.skrivervik@epfl.ch
}

\begin{abstract}
Fundamental limits for implanted antennas should be able to evaluate the bounds on losses with the knowledge of physical limitations from antenna and tissue parameters. In this work, rules of thumb to assess losses of deeply implanted antennas are presented toward improving the radiation efficiency. By means of rigorous and approximate approaches, the radiation efficiency of an elementary antenna within a spherical phantom is calculated as a function of multiple variables. According to the rules summarized subsequently, losses from different contributions may play a key role in specific situations, such as changes in the antenna type, phantom size, implant location and operating frequency.
\end{abstract}

Index Terms-implanted antennas, radiation efficiency, losses, fundamental limits.

\section{INTRODUCTION}

Since the development of implantable pacemakers in the 1950s, implantable electronics are becoming more acceptable and available in recent decades and offer breakthrough capabilities for a wide variety of medical diagnostics, treatments and therapies [1]-[3]. Today, more cutting-edge technologies allow the realization of implantable electronics with higher complexity, further miniaturization, etc. In order to achieve long-term operation of implants, wireless communication and powering through the lossy phantom have become an indispensable and challenging task, where the implanted antenna has become a crucial implantable electronic component [4]. Compared to increasing the transmitting power for communication or powering, a comprehensive understanding of implanted antennas is generally more economical, since the improvement of the implanted antenna in terms of radiation efficiency will do more with less power under the guidance of appropriate design rules.

In free space, physical bounds on radiation efficiency of arbitrary antennas have been extensively studied [5]-[8]. However, for implanted antennas, the main source of radiation losses is in general due to the dissipation and scattering in the lossy biological tissues and the level of miniaturization required. To analyze the basic radiation properties of implanted antennas, a simplified body model can be a good choice [9]-[16]. Although only approximate results can be obtained, they can provide more physical insights for implanted antennas, which will be beneficial to put forward the design rules. For instance, based on the spherical dyadic Green's function expansions and finitedifference time-domain code, Kim and Rahmat-Samii [9] analyzed the electromagnetic characteristics of dipole antennas and low-profile patch antennas implanted in the human head and body. Poon et al. [10] showed that the optimal frequency can be achieved at GHz-range for an inbody device operating in the midfield region. Using spherical wave expansion method, Merli et al. [11] studied the effect of implantable device encapsulation on radiation efficiency, which improves with increasing permittivity and thickness of encapsulation.

This paper aims to investigate the radiation efficiency of the deeply implanted antennas using canonical models, as was first proposed in [16]. Following this approach and using a spherical wave expansion (SWE) to estimate the radiation efficiency of implanted canonical antennas (elementary sources) [16]-[22], this paper presents the evolution of the radiation efficiency as a function of multiple variables: the frequency, the radius of the phantom and source, and the depth of the implant. Based on the understanding of the loss mechanisms in these canonical cases, rules of thumb to assess the losses of deeply implanted antennas are summarized qualitatively for different situations, e.g. changes in the antenna type, phantom size, implant location and operating frequency.

\section{MODEL AND ANALYSIS METHODS}

To analyze the losses of implanted antennas within the human body, we follow here the method developed in [22]: The antenna, an elementary electric or magnetic dipole, is implanted in a spherical homogeneous or stratified body phantom. The model is depicted in Fig. 1 for the case of a homogeneous phantom. The source is surrounded by a small lossless sphere, usually air, of radius $r_{\text {impl }}$ (roughly represents the antenna size). It is located at a distance $r_{\text {feed }}$ from the center of the spherical phantom, the latter having a radius $r_{\text {body. }}$

\section{A. Spherical Wave Expansion (SWE)}

Regarding the spherical body phantom, our first solution procedure makes use of the SWE proposed in [16]. Depending on the design of the model structure, the source can be placed at any point inside of the spherical phantom, 
and the phantom can be made stratified (representing additional fat/skin layers, for instance) with the help of a mode matching technique presented in [21].

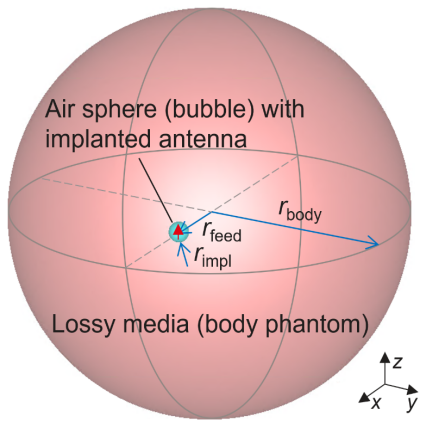

Fig. 1. View of the homogeneous spherical body phantom with the offcentered excitation.

In the SWE method presented in [16], for the case where an off-centered source is implanted in a spherical phantom, the structure of interest consists of two spherical structures, i.e the body model and the antenna model, which are connected using addition theorems. In this paper, a simplified method is adopted: the source is directly placed in the spherical phantom without the surrounding lossless sphere, and the calculation results are normalized by the corresponding value at the outermost point of the lossless sphere boundary. Since the radius of the antenna (i.e. the radius of the surrounding lossless sphere $r_{\text {impl }}$ ) is always relatively small, the field distribution at the its boundary will contain only the fundamental spherical mode. Therefore, according to the equivalence principle, such a simplified method is reasonable for most implanted antennas with small radius (i.e. $r_{\text {impl }} \ll \lambda$ ).

\section{B. Approximations of the Radiation Efficiency for Implanted Antennas}

When the source is placed at the center of a homogeneous spherical phantom, approximations of the radiation efficiency for implanted antennas were proposed in [22]. Here only the final expressions are repeated for the case with an electric dipole as excitation. The total radiated power reaching free-space could be expressed as

$$
P_{\text {total radiated power }}=P_{\text {entering the body }} \cdot e_{\text {near field }} \cdot e_{\text {propagating field }} \cdot e_{\text {reflections }}
$$

In this way, we calculate the radiation efficiency as the ratio of $P_{\text {total radiated power }}$ to $P_{\text {entering the body. }}$

The specific expressions for losses due to these different contributions are expressed as

$$
e_{\text {near field }}=\frac{|k|^{2} \operatorname{Re}(\eta)}{\operatorname{Re}\left\{\eta\left[|k|^{2}+2 \alpha r_{\text {impl }}^{-1}+\left(1-\frac{k^{*}}{k}\right) r_{\text {impl }}^{-2}-\mathrm{j} k^{-1} r_{\text {impl }}^{-3}\right]\right\}}
$$

$$
e_{\text {propagating field }}=e^{-2 \alpha\left(r_{\text {body }}-r_{\text {impl }}\right)},
$$

$$
e_{\text {reflections }}=\frac{\operatorname{Re}\left(|T|^{2} / Z_{\text {air }}\right)}{\operatorname{Re}\left(1 / Z_{\text {body }}\right)}, T=\frac{2 Z_{\text {air }}}{Z_{\text {air }}+Z_{\text {body }}},
$$

where $\eta$ is the wave impedance in the considered medium, $k$ denotes the complex wave number in this medium, and $\alpha$ is the real part of the propagation constant. $Z_{\text {air }}$ and $Z_{\text {body }}$ are the mode impedances of the spherical wave in the corresponding medium.

Compared with SWE, this approximation helps to understand and separate the contribution of losses in different situations, which will guide the design of the antenna to work on the primary one in order to improve the radiation efficiency.

\section{RESULTS}

Our first example is similar to the implant described in [12]. However, we use here a Hertzian dipole as an antenna. We consider an electric dipole surrounded by a bubble having a radius $r_{\mathrm{impl}}=5 \mathrm{~mm}$ implanted at the center point of a spherical body phantom with the radius $r_{\text {body }}=10 \mathrm{~cm}$. The phantom is homogeneously composed of muscle tissue, and its permittivity is provided by the four-region Cole-Cole model defined in [23]. Fig. 2 shows the radiation efficiency as a function of the frequency (from $100 \mathrm{MHz}$ to $4 \mathrm{GHz}$ ) computed using SWE and the approximations of the radiation efficiency for implanted antennas, respectively. The results using two different methods are almost superimposed, and these results correspond well to the ones published in [12] (see Fig. 2(d) in [12]), although the sources in the latter work are shaped as cylindrical capsule antennas with similar dimensions.

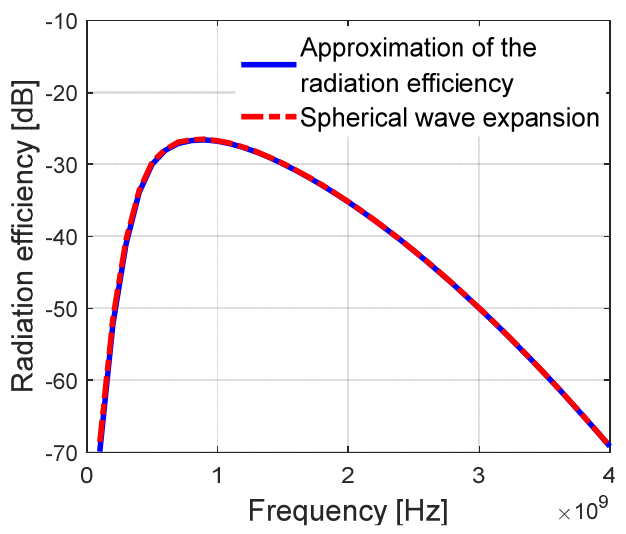

Fig. 2. Spectrum of the radiation efficiency excited by an electric dipole placed at the center point of the spherical body phantom.

Using the same methods, we investigated the radiation efficiency for the same canonical case as a function of the frequency and the radius of the phantom $r_{\text {body. The source is }}$ still placed at the center point of a spherical phantom made 
of muscle, and $r_{\text {impl }}$ is kept at $5 \mathrm{~mm}$. Electric and magnetic dipoles are considered. The results are shown in Fig. 3 for the electric dipole and in Fig. 4 for the magnetic dipole. The results computed via the approximations of the radiation efficiency for implanted antennas [Fig. 3 (a) and Fig. 4(a)] are very similar to the results by the SWE [Fig. 3 (b) and Fig. 4 (b)]. Deviations can be found when the radius of the phantom $r_{\text {body }}$ is small. This happens when near-field losses and the losses due to reflections at the phantom-air interface are predominant.

According to Fig. 3, the following insight can be gained:

- The frequency and $r_{\text {body }}$ correlatedly affect the value of the radiation efficiency. This is mainly because the frequency and the properties of the phantom (i.e. its size and composition) jointly determine the field region of the source where the phantom-air interface is located. Another important factor is that the permittivity of the lossy tissues, like muscle, is always a function of the frequency (especially, the imaginary part of permittivity that represents losses due to both displacement and ionic currents).

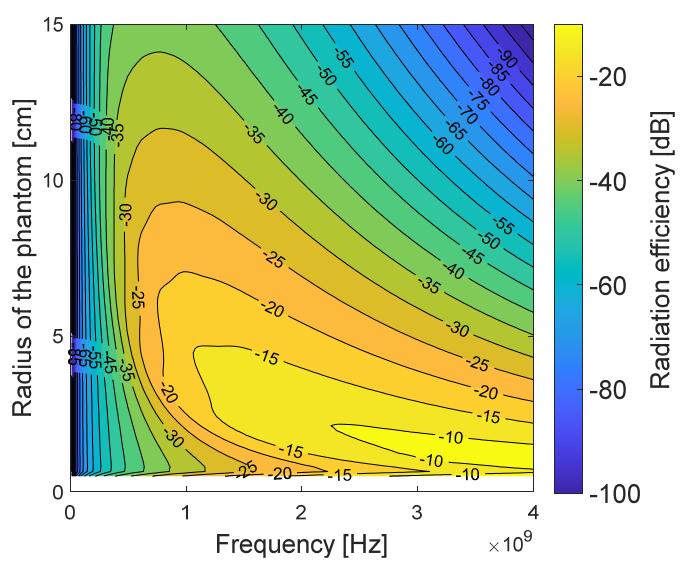

(a)

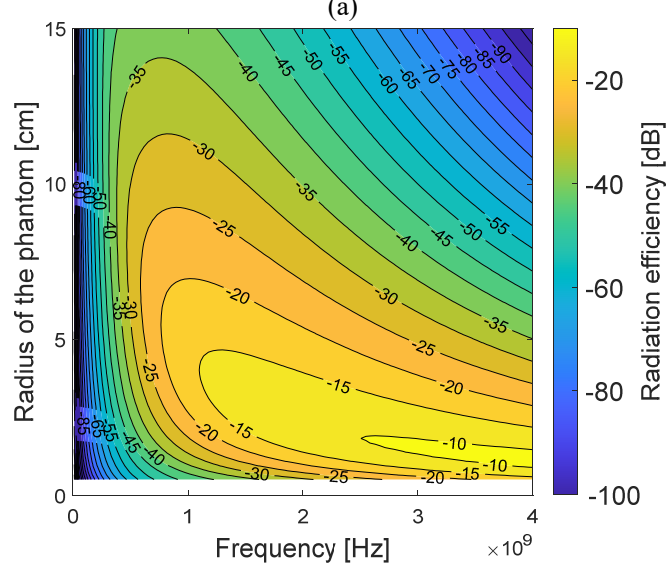

(b)

Fig. 3. Distributions of the radiation efficiency for an electric dipole as a function of the frequency and the radius of the phantom $r_{\text {body }}$ via (a) the SWE and (b) the approximations of the radiation efficiency for implanted antennas.

- For low frequencies (e.g. $\lesssim 500 \mathrm{MHz}$ in the case of electric dipole), losses due to the reactive near-field absorption will significantly decrease radiation efficiency; when the frequency increases, losses due to propagating field absorption begin to play a major role in reducing the radiation efficiency.

- When the radius of the phantom $r_{\text {body }}$ is small, the contribution of reflections losses can't be ignored; as $r_{\text {body }}$ increases, the losses due to propagating field absorption become more dominant.

- For the case of electric dipole implanted at the center point of the spherical body phantom with $r_{\text {body }}$ of several centimeters, the frequency that optimizes the radiation efficiency is in the GHz-range or sub-GHz range. This being said, we see that, as $r_{\text {body }}$ increases, the satisfactory values of this frequency start to decrease, but its descending speed will gradually slow down, as in this case when $r_{\text {body }}$ is larger than $5 \mathrm{~cm}$.

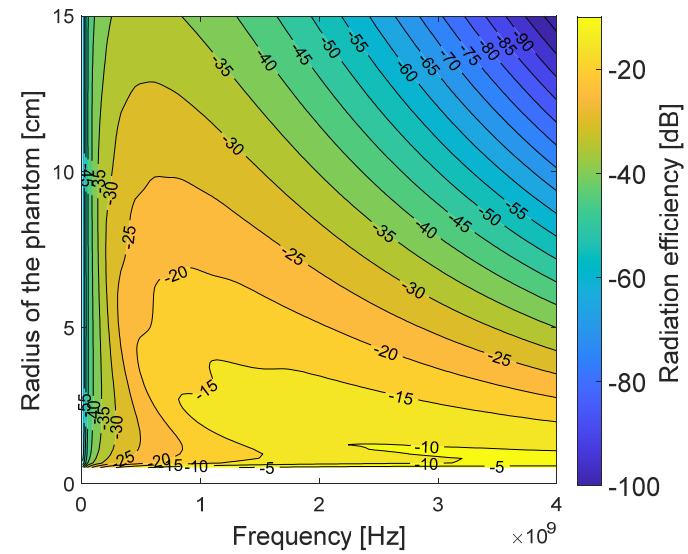

(a)

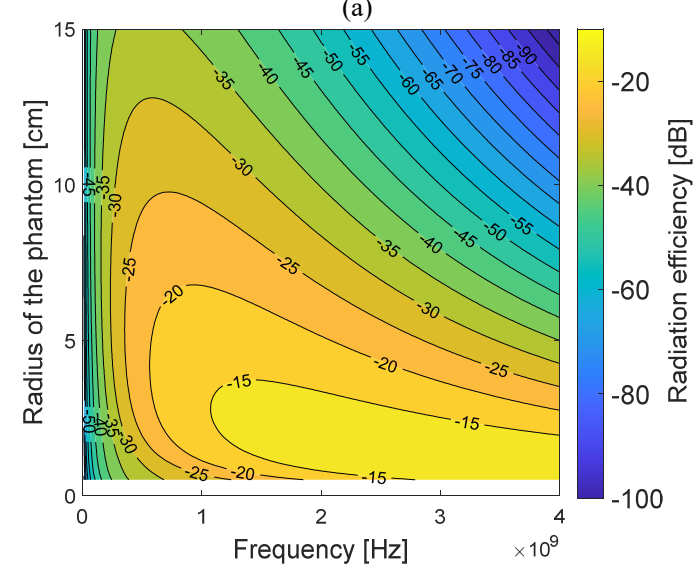

(b)

Fig. 4. Distributions of the radiation efficiency for a magnetic dipole as a function of the frequency and the radius of the phantom $r_{\text {body }}$ via (a) the SWE and (b) the approximations of the radiation efficiency for implanted antennas.

Similarly, the above analyses are mostly applicable to the case excited by a magnetic dipole, as shown in Fig. 4 (a) and (b). Some more insight can be obtained:

- Compared with the results of an electric dipole, the effect of the near-field losses produced by a magnetic dipole is significantly smaller. Therefore, we see that the optimal frequency regarding the radiation 
efficiency becomes lower than in the case of the electric dipole case, especially as $r_{\text {body }}$ increases to more than $5 \mathrm{~cm}$.

The radius of the implanted antenna $r_{\text {impl }}$ is another key parameter for implanted antennas. Fig. 5 (a) and (b) show the distributions of the radiation efficiency as a function of the frequency and $r_{\text {impl }}$ for an electric and a magnetic dipole placed at the center point, respectively, in which $r_{\text {body }}$ is fixed to $5 \mathrm{~cm}$. Comparing these results, we could find that:

- Whether for the implanted electric dipole or magnetic dipole, the increase in $r_{\text {impl }}$ reduces the losses especially the reactive near-field losses. Since the near-field losses for the electric dipole are relatively large, the frequency that optimizes the radiation efficiency start to increase when $r_{\text {impl }}$ becomes small enough [i.e. $r_{\text {impl }}<5 \mathrm{~mm}$ in Fig. 5 (a)]. However, for the magnetic dipole, the acceptable range of the optimal frequency does not change much with the decrease of $r_{\text {impl }}$.

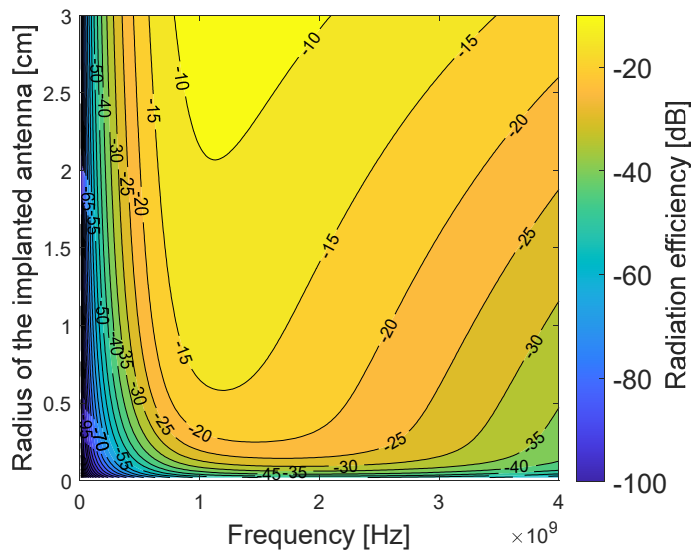

(a)

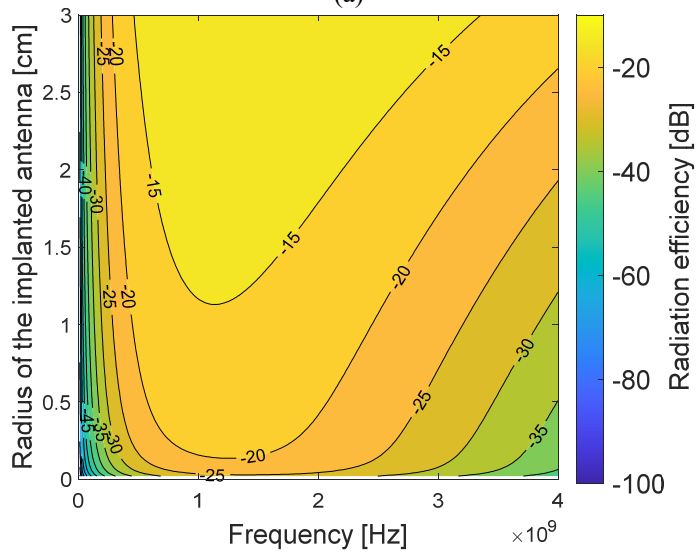

(b)

Fig. 5. Distributions of the radiation efficiency for (a) an electric dipole and (b) a magnetic dipole as a function of the frequency and the radius of the implanted antenna $r_{\text {impl, }}$ computed using the approximation of the radiation efficiency for implanted antennas.

Finally, by the SWE, a more general case has also been investigated: an electric dipole deeply implanted in a spherical phantom made of muscle $\left(r_{\text {body }}=10 \mathrm{~cm}\right)$ with the offset distance $r_{\text {feed }}$ increasing from 0 to $5 \mathrm{~cm}$. We notice that:

- When $r_{\text {feed }}$ increases to a relatively small length (i.e. smaller than $4 \mathrm{~cm}$ in this case), the frequency that optimizes the radiation efficiency is basically maintained in a fixed range under the tolerance of -1 $\mathrm{dB}$, as shown in Fig. 6. This means that the rules and conclusions obtained before are also applicable to most deeply implanted antennas, despite they may not be placed at the center point of the spherical phantom.

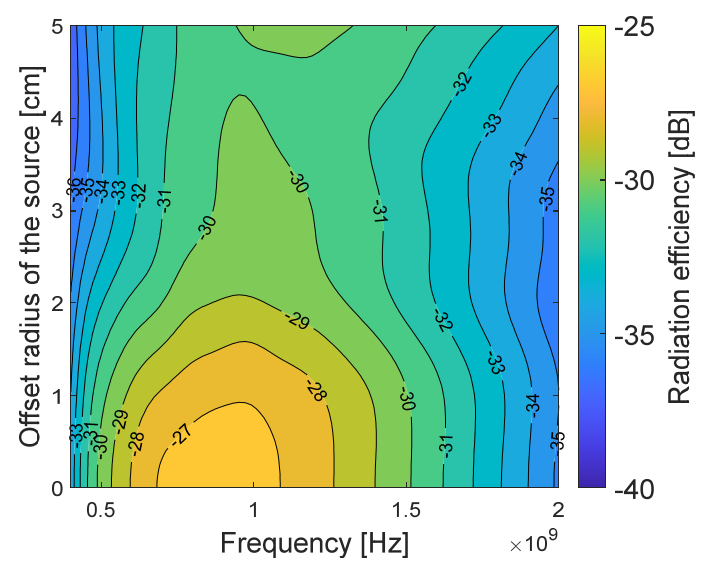

Fig. 6. Distributions of the radiation efficiency for an electric dipole as a function of the frequency and the offset radius of the source.

\section{CONCLUSION}

In this paper, we have investigated the radiation efficiency of an antenna deeply implanted in a lossy phantom using canonical models. According to the results for different situations, rules of thumb to assess losses of implanted antennas have been presented. We summarized the effects of frequency and phantom radius on the losses due to three contributions, i.e. reactive near-field absorption, propagating field absorption and reflections. We then analyzed the optimal frequency (the frequency yielding the highest overall radiation efficiency) and the according radiation efficiency as a function of the type of the source (electric or magnetic), the size of the phantom, the depth of the implant and the size of the implant. The established guidelines facilitate the choice of the antenna type and dimensions and provide simple rules to check the feasibility of a given antenna specification.

\section{ACKNOWLEDGMENT}

This work was supported in part by the China Scholarship Council.

\section{REFERENCES}

[1] D. Fitzpatrick, Implantable Electronic Medical Devices. New York, NY, USA: Elsevier, 2014.

[2] Y. Song, J. Min, W. Gao. "Wearable and implantable electronics: moving toward precision therapy," ACS nano, vol 13, no. 11, pp. 12280-12286, 2019. 
[3] H. Dinis, I. Colmiais and P. M. Mendes, "Extending the limits of wireless power transfer to miniaturized implantable electronic devices", Micromachines, vol. 8, no. 12, pp. 359, 2017.

[4] A. K. Skrivervik, "Implantable antennas: The challenge of efficiency", in Proc. $7^{\text {th }}$ Eur. Conf. Antennas Propag. (EuCAP 2013), Gothenburg, Sweden, pp. 3516-3520, 2013.

[5] H. A. Wheeler, "Fundamental limitations of small antennas," Proc. IRE, vol. 35, no. 12, pp. 1479-1484, Dec. 1947.

[6] L.J. Chu, "Physical limitations of omni-directional antennas", J. Appl. Phys., vol. 19, no. 12, pp. 1163-1175, 1948.

[7] R.F. Harrington, "On the gain and beamwidth of directional antennas", IRE Trans. on Antennas and Propag., vol. AP-6, 1958, pp. 219-225.

[8] M. Gustafsson, C. Sohl and G. Kristensson, "Physical limitations on antennas of arbitrary shape", Proc. R. Soc. A, vol. 463, pp. 25892607, 2007.

[9] J. Kim and Y. Rahmat-Samii, "Implanted antennas inside a human body: Simulations, designs, and characterizations," IEEE Trans. Microw. Theory Tech., vol. 52, no. 8, pp. 1934-1943, Aug. 2004.

[10] A. S. Y. Poon, S. O. Driscoll, and T. H. Meng, "Optimal frequency for wireless power transmission into dispersive tissue," IEEE Trans. Antennas Propag., vol. 58, no. 5, pp. 1739-1750, 2010.

[11] F. Merli, B. Fuchs, J. R. Mosig, and A. K. Skrivervik, "The effect of insulating layers on the performance of implanted antennas," IEEE Trans. Antennas Propag., vol. AP-59, no. 1, pp. 21-31, Jan. 2011.

[12] D. Nikolayev, W. Joseph, M. Zhadobov, R. Sauleau, and L. Martens, "Optimal radiation of body-implanted capsules," Phys. Rev. Lett., vol. 122, no. 10, p. 108101, Mar. 2019.

[13] D. Nikolayev et al., "Electromagnetic radiation efficiency of bodyimplanted devices," Phys. Rev. Appl., vol. 9, no. 2, p. 024033, Feb. 2018.
[14] D. P. Chrissoulidis and J.-M. Laheurte, "Radiation from an encapsulated Hertz dipole implanted in a human torso model," IEEE Trans. Antennas Propag., vol. 64, no. 12, pp. 4984-4992, Dec. 2016.

[15] Z. Sipus, M. Bosiljevac, D. Nikolayev and A. Skrivervik, "Design concerns for in-body antennas based on frequency analysis of fundamental radiation limitations," in Proc. $14^{\text {th }}$ Eur. Conf. Antennas Propag. (EuCAP 2020), Copenhagen, Denmark, 2020, pp. 1-5.

[16] M. Bosiljevac, Z. Sipus, and A. K. Skrivervik, "Propagation in finite lossy media: An application to WBAN," IEEE Antenn. Wireless Propag. Lett., vol. 14, pp. 1546-1549, 2015.

[17] J. A. Stratton, Electromagnetic Theory. New York, NY, USA: McGraw-Hill, 1941.

[18] R. F. Harrington, Time-Harmonic Electromagnetic Fields. New York, NY, USA: McGraw-Hill, 1961.

[19] S. Stein, "Addition theorems for spherical wave functions," Quart.Appl. Math., vol. 19, pp. 15-24, 1961.

[20] W. C. Chew, Waves and Fields in Inhomogenous Media. Piscataway, NJ, USA: IEEE Press, 1995.

[21] B. Fuchs, S. Palud, L. L. Coq, O. Lafond, M. Himdi, and S. Rondineau, "Scattering of spherically and hemispherically stratified lenses fed by any real source," IEEE Trans. Antennas Propag., vol. 56, no. 2, pp. 450-460, Feb. 2008.

[22] A. Skrivervik, M. Bosiljevac, Z. Šipuš, "Fundamental limits for implanted antennas: maximum power density reaching free space", IEEE Trans. Antennas Propag., vol. 67, no. 8, pp. 4978-4988, Aug. 2019.

[23] S. Gabriel, R.W. Lau, and C. Gabriel, "The dielectric properties of biological tissues: III. Parametric models for the dielectric spectrum of tissues," Phys. Med. Biol., vol. 41, 2271, 1996. 